Tage 60, 40 und $20 \mathrm{mg}$ ), 88 erhielten ein Placebo. Der Funktionsstatus gemäß ODI hatte sich nach drei Wochen in beiden Gruppen signifikant gebessert, von 51,2 auf 32,3 Punkte mit Prednison und von 51,1 auf 37,5 Punkte mit Placebo. Nach statistischer Bereinigung ergab dies eine um 6,4 Punkte größere Reduktion in der Steroidgruppe. Die Chance auf einen Rückgang des ODI-Scores um mindestens 30 Punkte wurde durch das Steroid um $70 \%$ erhöht (NNT: 10,6).

Der Vorteil der Verumgruppe im ODI war nach sechs Wochen verschwunden, überraschenderweise nach einem Jahr aber wieder feststellbar. Die Autoren sehen dies als Zufallsprodukt, da ein ver- zögerter Effekt mit der Wirkungsweise des Steroids kaum zu erklären ist.

Der Summenscore für die physische Komponente des SF-36 hatte sich nach drei Wochen ebenfalls in der Prednisongruppe stärker gebessert. Nach 52 Wochen war hier kein Unterschied mehr zu erkennen.

Keinen Einfluss hatte die Steroidtherapie auf die in die Beine ausstrahlenden Schmerzen. Sie waren in beiden Gruppen nach drei Wochen und nach einem Jahr gleichermaßen zurückgegangen. Außerdem unterzog sich fast jeder zehnte Patient im Lauf des Jahres einer Wirbelsäulenoperation - unabhängig davon, welcher Gruppe er angehörte.

\title{
Lokalanästhesie: Kein Vorteil bei Hüft-TEP mit anteriorem Zugang
}

\begin{abstract}
Weder die Standardlokalanästhesie noch eine reverse Infiltration können bei einer Hüft-Totalendoprothese (TEP) mit anteriorem Zugang den postoperativen Schmerz verbessern. Zu diesem Ergebnis kam eine niederländische Studie beim Vergleich beider Methoden mit Placebo.
\end{abstract}

$Z^{\text {un }}$ um multimodalen Schmerzmanagement einer Hüftendoprothetik gehört heute häufig eine Infiltrationsanästhesie im Gewebe um das Operationsfeld. Eine gute Schmerzkontrolle soll gewährleisten, dass der Patienten nach der TEP schnell wieder auf den Beinen ist.

In einer prospektiven, randomisierten und placebokontrollierten Studie haben Yvon den Hartog et al. vom Reinier de Graaf Hospital in Delft bei Hüft-TEP mit anteriorem Zugang die Standardinfiltration eines Lokalanästhetikums (subkutane Infiltration kurz vor Wundschluss) mit einer reversen Infiltrationsmethode („reverse infiltration“ von Ropivacain 2 $\mathrm{mg} / \mathrm{ml}$ und Epinephrin $1 \mathrm{mg} / \mathrm{ml} \mathrm{im}$ Verhältnis $99: 1$, beginnt mit der subkutanen Infiltration noch vor dem ersten Schnitt) sowie Placebo $(0,9 \% \mathrm{NaCl})$ verglichen. Ansonsten erhielten alle Patienten die gleiche Behandlung. Alle Eingriffe wurden von demselben Chirurgen ausgeführt. Primärer Endpunkt der Studie war die Schmerzstärke vier Stunden nach der Operation auf einer numerischen Skala von 0 bis 10 (NRS). Außer- dem wurden die Länge des Klinikaufenthalts, die Operationsdauer, der Zielort des Patienten nach der Entlassung, der Schmerzmittelverbrauch, Nebenwirkungen sowie Schmerz-Scores zu verschiedenen weiteren Zeiten nach dem Eingriff berücksichtigt.

Zwischen November 2012 und Januar 2014 wurden 75 Probanden im Durchschnittsalter von 67,1 Jahren mit Hüftarthrose in die Studie eingeschlossen und in die drei Gruppen randomisiert. $74 \mathrm{~Pa}$ tienten erhielten eine zementfreie Prothese, ein Patient mit schwerer Osteoporose eine zementierte Hüft-TEP. Vier Stunden nach dem Eingriff konnten hinsichtlich des mittleren Schmerz-Scores keine Unterschiede zwischen den Gruppen festgestellt werden. Der numerische Score ergab Werte von 3,1 beziehungsweise 2,9 beziehungsweise 2,9 für die Schmerzstärke. Nach einer und nach acht Stunden zeigten sich postoperativ in der Placebogruppe zwar signifikant mehr Nebenwirkungen (z. B. Schwindel, Hypotonie, Harnverhalt, Kopfschmerz), diese beeinflussten die Mobilisation der
Fazit: Kann die mit dem Steroid erzielte Veränderung im ODI von 6,4 Punkten auf einer 100-Punkte-Skala eine Behandlung begründen? Die minimale Differenz im ODI, die als klinisch relevant angesehen wird, variiert in der Literatur zwischen 5 und 15 Punkten. „Ob die beobachtete Funktionsverbesserung den Gebrauch oraler Steroide rechtfertigt, ist eine schwierige Entscheidung, die letztlich individuell von Arzt und $\mathrm{Pa}$ tienten abgewogen werden muss", so die Autoren.

Beate Schumacher

Goldberg $\mathrm{H}$ et al. Oral steroids for acute radiculopathy due to a herniated lumbar disk: a randomized clinical trial. JAMA 2015; 313 (19): 1915-23

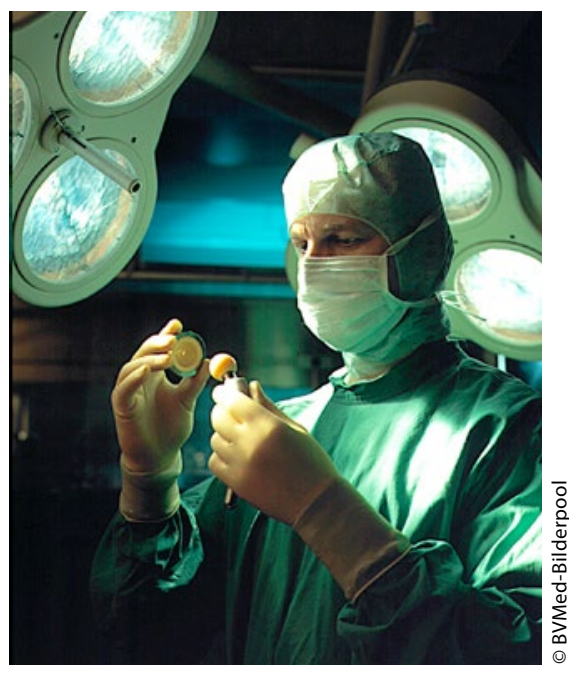

Lokalanästhesie bringt bei Hüft-TEP mit anteriorem Zugang keinen Vorteil

Patienten aber nicht. Auch bei allen anderen Parametern ergaben sich in allen drei Gruppen ähnliche Ergebnisse.

Fazit: Im Rahmen eines multimodalen Schmerzkonzeptes bringt die Lokalanästhesie mit Ropivacain und Epinephrin bei der Hüft-TEP mit anteriorem $\mathrm{Zu}$ gang den Studienautoren zufolge offenbar keinen zusätzlichen Nutzen.

\section{Dr. Christine Starostzik}

den Hartog YM et al. No effect of the infiltration of local anaesthetic for total hip arthroplasty using an anterior approach. A randomised placebo controlled trial. Bone Joint J 2015; 97B:734-40; doi: 10.1302/0301-620X.97B6.35343 